\title{
Sleep Selectively Enhances Memory Expected to Be of Future Relevance
}

\author{
Ines Wilhelm, ${ }^{1}$ Susanne Diekelmann, ${ }^{1}$ Ina Molzow, ${ }^{2}$ Amr Ayoub, ${ }^{1}$ Matthias Mölle, ${ }^{1}$ and Jan Born ${ }^{3}$ \\ ${ }^{1}$ Department of Neuroendocrinology, University of Lübeck, 23538 Lübeck, Germany, ${ }^{2}$ Center for Integrative Psychiatry, 24105 Kiel, Germany, and ${ }^{3}$ Institute \\ of Medical Psychology and Behavioral Neurobiology, University of Tübingen, 72074 Tübingen, Germany
}

The brain encodes huge amounts of information, but only a small fraction is stored for a longer time. There is now compelling evidence that the long-term storage of memories preferentially occurs during sleep. However, the factors mediating the selectivity of sleepassociated memory consolidation are poorly understood. Here, we show that the mere expectancy that a memory will be used in a future test determines whether or not sleep significantly benefits consolidation of this memory. Human subjects learned declarative memories (word paired associates) before retention periods of sleep or wakefulness. Postlearning sleep compared with wakefulness produced a strong improvement at delayed retrieval only if the subjects had been informed about the retrieval test after the learning period. If they had not been informed, retrieval after retention sleep did not differ from that after the wake retention interval. Retention during the wake intervals was not affected by retrieval expectancy. Retrieval expectancy also enhanced sleep-associated consolidation of visuospatial (two-dimensional object location task) and procedural motor memories (finger sequence tapping). Subjects expecting the retrieval displayed a robust increase in slow oscillation activity and sleep spindle count during postlearning slow-wave sleep (SWS). Sleepassociated consolidation of declarative memory was strongly correlated to slow oscillation activity and spindle count, but only if the subjects expected the retrieval test. In conclusion, our work shows that sleep preferentially benefits consolidation of memories that are relevant for future behavior, presumably through a SWS-dependent reprocessing of these memories.

\section{Introduction}

Not every event that is experienced during the wake period becomes stored for a long time. Rather, the brain appears to encode memories temporarily before it transfers some of them into a long-term storage system. The consolidation of memory, i.e., the transformation of a newly encoded memory into an endurable representation, has been suspected for some time to be particularly supported by sleep (Heine, 1914). Whereas classical theories explain this memory function of sleep as a permissive action whereby previously encoded memories during sleep are protected from interference and decay in a nonspecific manner (Jenkins and Dallenbach, 1924; Wixted, 2004), recent research has provided convergent evidence for active processes of consolidation present during sleep (Ellenbogen et al., 2006; Marshall and Born, 2007; Diekelmann and Born, 2010). Specifically, it was found that neuronal representations of hippocampus-dependent declarative memories are reactivated during slow-wave sleep (SWS) after learning and that these reactivations cause the consolidation of respective memories (Wilson and McNaughton, 1994; Peigneux et al., 2004; Ji and Wilson, 2007; Rasch et al., 2007), possibly by supporting a redistribution of the representations from hippocampal to neocortical sites for long-term storage

Received July 10, 2010; revised Nov. 10, 2010; accepted Nov. 21, 2010.

This work was supported by the Deutsche Forschungsgemeinschaft (Sonderforschungsbereich 654). We thank Isabelle Schlüter and Julia Niederquell for technical assistance and Sabine Groch and Lisa Marshall for comments on a previous version of the manuscript.

Correspondence should be addressed to Jan Born, Institute of Medical Psychology and Behavioral Neurobiology, University of Tübingen, Gartenstrasse 29,72074 Tübingen, Germany. E-mail: born@kfg.uni-luebeck.de.

DOI:10.1523/JNEUROSCI.3575-10.2011

Copyright $\odot 2011$ the authors $\quad 0270-6474 / 11 / 311563-07 \$ 15.00 / 0$
(Gais et al., 2007; Diekelmann and Born, 2010). However, the concept of an active system consolidation taking place during sleep implicates selectivity, i.e., that sleep preferentially enhances some memories over others, because the global strengthening of newly acquired memory traces and underlying synaptic connections by processes of reactivation would inevitably result in a system overflow. Yet, evidence for selectivity in sleep-dependent memory consolidation is presently scarce.

It is well known that the formation of memories is enhanced for information that is emotional and/or is of relevance for the formation of an individual's expectancies and future plans (Goschke and Kuhl, 1993; McGaugh, 2004; Badets et al., 2006). In 1927, Bluma Zeigarnik was the first to demonstrate that information associated with a planned action is better retained in memory as long as the plan is not executed (Zeigarnik, 1927). Since then, the driving role of future plans and expectancies for maintaining memories has been consistently confirmed in numerous studies (Shimizu, 1996; Marsh et al., 1998; Szpunar et al., 2007). However, because in these studies expectancies and plans were present already at learning, effects on consolidation could not be distinguished from those on encoding. Here, we provide novel evidence that simply informing subjects after a learning phase that newly acquired material will be needed in a future retrieval test critically increases the access of this memory to sleep-dependent memory consolidation.

\section{Materials and Methods}

Participants. A total of 142 healthy adults (18-35 years, mean \pm SD: $22.78 \pm 3.81$ years; 82 women) participated in experiment 1 , and 49 different healthy adults (mean \pm SD: $23.16 \pm 3.43$ years; 19 women) 
participated in experiment 2 (see Table S1, available at www.jneurosci. org as supplemental material, for detailed information on subject characteristics in the different groups of both experiments). The subjects were recruited via advertisements placed at the university. Subjects reported no history of any neurological or psychiatric disorder, no medication at the time of the experiments, no night shifts for at least 8 weeks before the experiment, and a regular sleep-wake cycle. On experimental days, they were not allowed to take in caffeine and alcohol or to nap during daytime. None of the subjects had previously participated in an experiment in our laboratory, and no subject participated in both experiments. All subjects of the sleep groups were habituated to polysomnographic recordings by spending an adaptation night in the laboratory that preceded the experiment proper by at least $2 \mathrm{~d}$. The study was approved by the local ethics committee, and subjects gave written informed consent before participating.

Design and procedures. In experiment 1 , subjects were assigned to one of six experimental groups (see Fig. $1 B$ for the experimental design). Subjects in each group learned a word paired-associate task in the evening (22:00-23:00 h) or in the morning (08:00-09:00 h), and half of the groups ("expected") were then informed that recall would be tested after a $9 \mathrm{~h}$ retention interval (i.e., at 08:00 or 18:00 h), whereas the other groups were instructed that they would perform on a different task at the second session ("unexpected"). Of the expected and unexpected groups, one was retested after a period of nocturnal sleep (final $n=18$ and 28, respectively), the second was retested after a period of daytime wakefulness ( $n=15$ and 32), and the third was retested after a period of nighttime wakefulness $(n=19$ and 30). Subjects of the unexpected groups who suspected the retrieval test were discarded from the main analyses ( $n=10,17$, and 11, for the sleep, daytime wake, and nighttime wake retention conditions, respectively) and were treated as separate "suspected" groups in additional analyses.

Sessions started with a short interview regarding health status, caffeine intake, etc. and, in the participants of the sleep groups, with preparations for polysomnographic recordings. Then, the participants filled out a questionnaire regarding feelings of tiredness, concentration, and motivation, followed by the learning phase. To exclude active rehearsal, subjects played the computer game Snood for $15 \mathrm{~min}$ after the end of the learning phase, i.e., immediately after subjects of the expected group had been instructed about the delayed retest. In the sleep conditions, subjects then immediately went to bed to allow sleep until 07:00 h. Retrieval was tested 60 min later. During wake retention intervals, subjects watched emotionally nonarousing films and played card and board games with the experimenter. After retrieval testing, the participants filled in a second questionnaire that included two questions as to whether they had engaged in any rehearsal of the materials acquired in the learning phase, i.e., "Did you think about the words after learning?" and "Did the words spontaneously come into your mind after learning?" (translated from German). Data from subjects affirming one of these questions were discarded from the analyses, although including them did not change the main findings of both experiments. Importantly, subjects of the unexpected groups were also asked whether or not they had expected the retrieval test after the retention interval. In case of an affirmative answer, data from respective subjects were used to form the separate suspected groups (see above).

In experiment 2, subjects were randomly assigned to two groups, an expected (final, $n=21$ ) and an unexpected group ( $n=27,10$ of which were excluded because they suspected the retrieval test). The procedures were practically the same as in the sleep conditions of experiment 1 except that two different memory tasks were learned, the two-dimensional (2D) object location task (applied first) and the finger sequence tapping task (applied second). Subjects of the expected group were informed about the later retrieval test after completion of both tasks. Recall was tested (in the same task order) after the retention interval and another time, a surprise for all subjects, 30 min later. After this surprise recall, subjects were again asked whether they had expected to be tested this second time, which was the case in two subjects of the expected group and in one subject of the unexpected group. In this experiment, additionally salivary cortisol was sampled shortly before learning and retrieval to exclude any confounding influence of stress on memory performance (for detailed results, see supplemental material, available at www.jneurosci.org).

Memory tasks. The three tasks employed in the two experiments have been proven to be sensitive to sleep-dependent memory consolidation in previous studies (Plihal and Born, 1997; Rasch et al., 2007; Walker et al., 2002). In experiment 1 , a word paired-associate learning task was used to test declarative memory. It required the subjects to learn a list of 40 pairs of semantically related words. During the learning phase, the word pairs were presented sequentially on a computer screen, each for $5 \mathrm{~s}$, separated by an interstimulus interval of $100 \mathrm{~ms}$. After presentation of the entire list, performance was tested using a cued recall procedure, i.e., the first word (cue) of each pair was presented and the subject had to name the associated second word (response). The correct response word was subsequently displayed for $2 \mathrm{~s}$, regardless of whether the response was correct or not, to allow re-encoding of the correct word pair. The cued recall procedure was repeated until the subject reached a criterion of $60 \%$ correct responses. Importantly, it should be noted that because of feedback and re-encoding in the final learning trial, learning performance does not reflect an accurate measure of immediate post-training memory performance. Consequently, the increase in recall at retest does not mean that there was an actual improvement in memory across the test-retest interval, but the sleep benefit as well as the benefit of expectancy presumably rather emerged in the form of diminished forgetting. Retrieval was tested using the same cued recall procedure as that used during the learning phase, except that the subjects did not receive feedback after the trials. Retention performance was defined by the percentage of recalled word pairs during retrieval, with performance on the final learning trial (criterion trial) set to $100 \%$.

In experiment 2 , all subjects first performed on a declarative $2 \mathrm{D}$ object location task and then on a procedural finger sequence tapping task, with the tasks separated by a 5 min break. The $2 \mathrm{D}$ object location task resembles the game Concentration and consists of 15 card pairs showing colored pictures of different animals and everyday objects. Throughout the task, all 30 possible spatial locations are shown as gray squares (representing the back of the cards) on a $15^{\prime \prime}$ screen. The locations are geometrically ordered in a $5 \times 6$ rectangular array. At learning, the first card of each card pair was presented alone for $1 \mathrm{~s}$ followed by the presentation of both cards for $3 \mathrm{~s}$. After an interstimulus interval of $3 \mathrm{~s}$, the next card pair was presented in the same way. The whole set of card pairs was presented twice, each time in a different order. Immediately after these two runs, recall of the spatial locations was tested using a cued recall procedure, i.e., the first card of each pair was presented and the subject had to indicate the location of the second card with a computer mouse. Visual feedback was given in each case by presenting the second card at the correct location for $2 \mathrm{~s}$. The cued recall procedure was repeated until the subjects reached a criterion of $60 \%$ correct responses, resulting in an individual task duration between 10 and 20 min. After presenting a card pair, both cards were replaced by gray squares again so that the guessing probability remained the same throughout each run. At retrieval testing the same cued recall procedure was used as during the learning phase, except that the subjects did not receive feedback after each trial. Retention performance was determined by the percentage of recalled card locations at retrieval, with performance on the criterion trial during learning set to $100 \%$.

The finger sequence tapping task requires the subject to press repeatedly a five element sequence (4-1-3-2-4) on a keyboard with the fingers of the nondominant hand as fast and as accurately as possible. The numeric sequence was displayed on the screen at all times to keep working memory demands at a minimum. Each key press resulted in a white dot in the center of the screen. At learning, subjects performed on twelve $30 \mathrm{~s}$ trials, each interrupted by $30 \mathrm{~s}$ breaks (overall task duration: $12 \mathrm{~min}$ ). Retrieval testing included three trials. To control for nonspecific changes in motor performance at retrieval testing, subjects subsequently performed on three trials of a new sequence not learned previously. For each $30 \mathrm{~s}$ trial the number of correctly tapped sequences was determined. Retention performance was determined by the average number of correctly tapped sequences across the three trials at retrieval, with the average performance on the last three trials at learning set to $100 \%$.

Sleep recordings, slow oscillation activity, and spindles. Standard polysomnographic recordings were obtained including EEG recordings from electrodes attached at C3 and C4 (according to the international 10-20 system, referenced to an electrode attached to the nose) and electrooculographic and electromyographic recordings. Signals were amplified 
(BrainAmp, Brain Products) and digitized, with the EEG sampled at a rate of $200 \mathrm{~Hz}$ and filtered between 0.16 and $70 \mathrm{~Hz}$. Sleep stages were determined offline for subsequent $30 \mathrm{~s}$ recording epochs following standard criteria (Rechtschaffen and Kales, 1968). Sleep onset (with reference to lights off), total sleep time, and the time of total sleep time spent in the different sleep stages [wake, stages 1,2,3 and 4, and rapid eye movement (REM) sleep] were determined. Slow-wave sleep was defined by the sum of time in stage 3 and 4 sleep. Latencies of SWS and REM sleep were assessed with reference to sleep onset, i.e., the first occurrence of a period of stage 1 sleep followed by stage 2 sleep.

For analyses of slow oscillation activity and spindles, subsequent 10.24 s (2048 data points) epochs of non-REM sleep (S2, S3, S4) were transformed using fast Fourier transform (Vision Analyzer, version 1, Brain Products). Only epochs free of movement or muscle artifacts (defined by EMG activity exceeding $\pm 50 \mu \mathrm{V}$ ) were used. Slow oscillation activity, defined by the mean power density within the $0.68-1.17 \mathrm{~Hz}$ frequency band, was averaged across six succeeding 20 min intervals of non-REM sleep occurring during the night, i.e., across $0-20,20-40,40-60,60-80$, $80-100,100-120 \mathrm{~min}$ of non-REM sleep. Note that the $20 \mathrm{~min}$ intervals also included noncontiguous periods, as they could be interrupted by periods of wakefulness, stage 1 , and REM sleep which were excluded. Data were then collapsed across both recording sites (C3, C4).

Spectrograms were also used to identify spindles in non-REM sleep stage 2 and SWS. Spindles were detected automatically using a custommade software tool (SpindleToolbox, version 3) that was based on an algorithm adopted from previous studies (Mölle et al., 2002). Briefly, first the power spectrum of each subject was calculated, enabling the user to visually detect the peak of the sigma frequency band in each individual. Then, the root mean square (rms) of the bandpass-filtered signal in the range $\pm 1.5 \mathrm{~Hz}$ around the detected spindle peak of each $200 \mathrm{~ms}$ interval was calculated, and the events in which the rms signal exceeded a constant threshold of $5 \mu \mathrm{V}$ for $0.5-3 \mathrm{~s}$ were counted.

\section{Results}

\section{Experiment 1}

The expectancy that recall would be tested after the retention interval significantly affected retention of the word pairs, not in the wake retention but only in the sleep retention condition $(p=$ 0.05 , for expectancy $\times$ sleep/wake interaction) $($ Fig. $1 A)$. When subjects slept after learning, the expectancy of a retrieval test after the retention interval led to a distinct improvement in recalled word pairs compared with performance of the unexpected group; i.e., the retention rate (indicated by the percentage of recalled word pairs at retrieval with performance on the criterion trial during learning set to $100 \%$ ) for the expected group was $117.29 \pm$ $3.54 \%$, and for the unexpected group it was $105.23 \pm 3.01 \%$ ( $p=$ $0.014)$. Retention performance in the expected subjects who had slept after learning was also superior to performance in all four wake retention groups (expected: daytime wakefulness was $104.89 \pm 3.76 \%$, nighttime wakefulness was $96.73 \pm 3.75 \%$; unexpected: daytime wakefulness was $101.33 \pm 3.48 \%$, nighttime wakefulness was $99.07 \pm 4.31 \%$; $p<0.025$ for all pairwise comparisons). Retention performance of the unexpected group with postlearning sleep was not significantly different from that of the unexpected wake groups ( $p>0.25$, for all pairwise comparisons). Also, informing subjects of the delayed recall did not improve word pair retention in the wake groups regardless of whether the wake retention interval occurred during daytime or nighttime ( $p>0.49$, for both comparisons). Interestingly, the subjects who were originally assigned to the unexpected groups, but whose data were discarded from the main analysis because they actually had suspected the retrieval test at the end of the retention interval, showed a similar pattern of retention performance as the subjects of the corresponding expected groups, with the retention rate after postlearning sleep (116.11 $\pm 4.40 \%)$ being significantly higher than that after the daytime $(102.20 \pm$
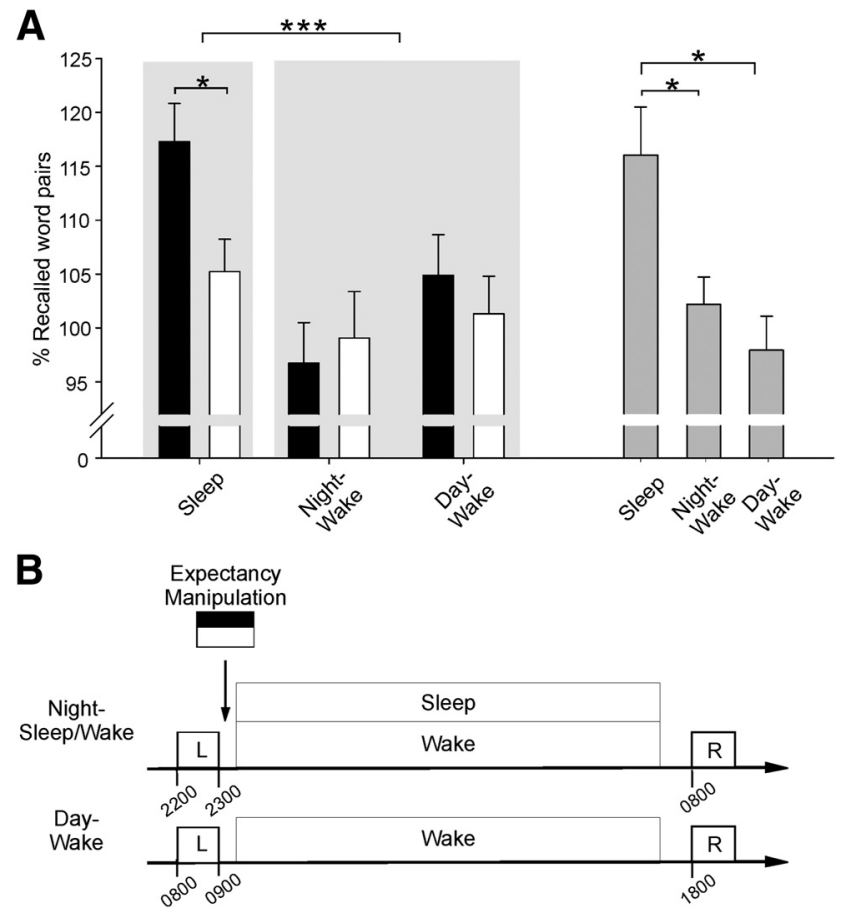

Figure 1. A, Effects of retrieval expectancy on retention of declarative memory for word pairs across $9 \mathrm{~h}$ intervals filled with nocturnal sleep (Sleep), nighttime wakefulness (Night-Wake), and daytime wakefulness (Day-Wake). Retention performance is indicated by the percentage of word pairs recalled at retrieval, with performance on the criterion trial during learning set to $100 \%$. Mean ( \pm SEM) retention performance is indicated for subjects who expected (expected group, black bars) or did not expect retrieval testing (unexpected group, open bars), as well as for subjects who suspected the retrieval although they were never explicitly informed about it, i.e., these subjects were originally assigned to the unexpected group (gray bars); ${ }^{*} p<0.05$, ${ }^{* * *} p<0.001$, for differences between groups. $\boldsymbol{B}$, Subjects in each group learned $(\mathrm{L})$ a pairedassociate task in the evening (22:00-23:00 h) or the morning $(08: 00-09: 00 \mathrm{~h})$, and half of the groups (expected) were then informed that recall (R) would be tested after a $9 \mathrm{~h}$ retention interval (i.e., at 08:00 or 18:00 h), whereas the other groups were not informed about the retrieval test (unexpected).

$2.48 \%)$ or nighttime wake intervals $(97.93 \pm 3.08 \%, p<0.015$, for both comparisons). Initial learning of the word pairs, as well as subjective ratings of tiredness, motivation, and concentration at learning and retrieval, was closely comparable in the six groups ( $p>0.10$ for all comparisons; see supplemental material for additional results from both experiments). Together, these data show that the benefit for word pair memories resulting from postlearning sleep is significant only if the subjects expected to be retested again on these memories.

Postlearning sleep in the subjects of the expected group contained on average more SWS with a significantly greater portion spent in the deepest sleep stage 4 than subjects of the unexpected group $(8.2 \pm 1.43 \%$ vs $4.07 \pm 1.16 \%, p=0.03$; see Table 1 for a summary of polysomnographic data). There were no differences between the groups for the other sleep parameters.

The increased amount of stage 4 sleep in the expected group was paralleled by a significant increase in EEG power in the slow oscillation frequency band $(0.68-1.17 \mathrm{~Hz})$ during the first $2 \mathrm{~h}$ of nocturnal non-rapid eye movement (non-REM) sleep in this group in comparison with the unexpected group $(p=0.025$; see also Table S2, available at www.jneurosci.org as supplemental material, for detailed information on the distribution of sleep stages in the first six 20 min intervals). Post hoc comparison analyses revealed that the third and the sixth 20 min interval significantly differed between the expected group and the unexpected 
Table 1. Sleep data

\begin{tabular}{lccccc}
\hline & \multicolumn{2}{l}{ Experiment 1 } & & \multicolumn{2}{l}{ Experiment 2 } \\
\cline { 2 - 3 } \cline { 5 - 6 } & \multicolumn{1}{l}{ Expected } & Unexpected & & Expected & \multicolumn{1}{c}{ Unexpected } \\
\hline Total sleep time & $446.79 \pm 6.40$ & $445.87 \pm 10.54$ & & $442.17 \pm 6.34$ & $454.40 \pm 5.32$ \\
Wake & $0.64 \pm 0.27$ & $1.67 \pm 0.88$ & & $1.82 \pm 0.71$ & $0.52 \pm 0.15$ \\
Stage 1 & $6.19 \pm 0.98$ & $5.37 \pm 0.71$ & & $6.07 \pm 1.95$ & $6.36 \pm 0.83$ \\
Stage 2 & $49.73 \pm 1.99$ & $53.41 \pm 2.38$ & & $49.66 \pm 2.99$ & $55.98 \pm 2.61$ \\
Stage 3 & $11.15 \pm 1.06$ & $11.66 \pm 0.65$ & & $9.79 \pm 0.96$ & $9.69 \pm 1.44$ \\
Stage 4 & $8.20 \pm 1.43$ & $4.07 \pm 1.16^{*}$ & & $11.72 \pm 1.81$ & $5.11 \pm 1.41^{* *}$ \\
SWS & $19.35 \pm 1.64$ & $15.73 \pm 1.55$ & & $21.51 \pm 2.49$ & $14.80 \pm 2.13$ \\
REM & $22.86 \pm 1.02$ & $22.84 \pm 1.60$ & & $20.00 \pm 1.89$ & $20.84 \pm 1.11$ \\
SWS latency & $18.68 \pm 2.10$ & $20.03 \pm 1.32$ & & $16.06 \pm 1.55$ & $18.15 \pm 2.43$ \\
REM latency & $88.64 \pm 9.38$ & $77.13 \pm 4.57$ & & $81.50 \pm 5.38$ & $90.45 \pm 10.40$ \\
\hline
\end{tabular}

Data for mean ( \pm SEM) total sleep time, time spent awake, stage 1 sleep, stage 2 sleep, stage 3 sleep, stage 4 sleep, slow wave sleep (the sum of stage 3 and 4 sleep) and REM sleep given in percentage of total sleep time, and latency of the first period of SWS and REM sleep (with reference to sleep onset) are shown; ${ }^{*} p<0.05$, ${ }^{* *} p<0.01$, for differences between groups.

group (Fig. 2A). Of note, both slow oscillation activity at the beginning of sleep (first $20 \mathrm{~min}$ of non-REM sleep) and the proportion of stage 4 SWS were significantly correlated with the retention of word pairs, but only in the expected group $(r=0.71$ and $r=0.71, p<0.01$ for both coefficients) (Fig. $2 B$ ). Respective correlations for the later 20 min intervals were smaller $(r<0.58)$ and not significant $(p>0.06)$. There were no such associations in the unexpected group ( $r=-0.25$ and $r=-0.36, p>0.19)$. Slow oscillation activity, as expected, was highly correlated with the amount of SWS $(r=0.95 ; p<0.001)$ indicating that both measures under natural conditions reflect closely related phenomena (although basically slow oscillation activity can be experimentally suppressed without significantly changing SWS) (van der Werf et al., 2009). Further analyses, performed to clarify the functional relevance of slow oscillation activity, revealed that the correlation of slow oscillation activity with memory performance was 0.56 ( $p=0.07$ ) when determined for the first 20 min of SWS $(\mathrm{S} 3, \mathrm{~S} 4)$ and $0.71(p=0.01)$ when determined for the first $20 \mathrm{~min}$ of non-REM sleep (including 14 min of stage 2 sleep), the latter suggesting a particular relevance of slow oscillation activity occurring early during sleep beyond the presence of SWS per se. Spindle count and density in sleep stage 2 as well as spindle density in SWS were not affected by the expectancy of being retested after sleep. Spindle count during SWS was on average higher in the expected than unexpected group ( $316 \pm 69$ vs $198 \pm 42)$, but this difference was not significant $(p=0.15)$.

\section{Experiment 2}

We aimed to extend the finding of a differential effect of retrieval expectancy in the sleep group of experiment 1 to different learning materials and memory systems, i.e., a declarative memory task which is nonverbal (the $2 \mathrm{D}$ object location task), and a procedural memory task (the finger sequence tapping task).

Like retention of word pairs in experiment 1, retention of card pair locations in the $2 \mathrm{D}$ object location task was distinctly enhanced across sleep if the subjects expected the retrieval test (expected group) in comparison with the unexpected group (percentage of recalled card pairs with reference to performance on the criterion trial at learning: $98.80 \pm 4.92$ vs $73.13 \pm 9.08 \%, p=$ 0.019 ) (Fig. $3 A$ ). Retention performance in the subjects who were excluded from the unexpected group because they had suspected the retrieval test $(89.67 \pm 7.09 \%)$ was also enhanced compared with performance in the unexpected group, although this increase failed to reach significance ( $p=0.17$ ).

The overnight gain in procedural finger sequence tapping skill was also greater for the expected group than the unexpected group (percentage improvement at retrieval with reference to performance at the end of the learning phase: $131.13 \pm 4.08 \%$ vs $116.95 \pm 5.04 \%, p=0.037$ ) (Fig. $3 B$ ), with the gain in the expected group appearing even slightly higher than those reported in comparable previous studies (e.g., Walker et al., 2003, 2005). Gains in skill in the subjects who were excluded from the unexpected group due to their suspecting the retrieval test were on average closely comparable to those of the expected group $(131.63 \pm 9.39 \%)$, but because of their greater variability these gains did not significantly differ from those of the unexpected group ( $p=0.15)$. Performance on a novel sequence introduced after retrieval testing was clearly worse than on the learned sequence $(p<0.001)$ with no difference between the groups $(p>0.93)$, indicating that the effect of retrieval expectancy was specific to the learned sequence.

It could be argued that the expectancy of a retrieval test does not improve retention performance by enhancing consolidation, but rather by an acute effect on retrieval processes. The expectancy present at the time of retrieval may nonspecifically prime memory networks and thereby ease the retrieval of relevant materials. In this case, differences in recall should disappear when the retrieval test comes as a surprise for both groups. To explore this possibility, we tested retrieval of card pair locations in all subjects unexpectedly a second time $30 \mathrm{~min}$ later. We found that recall performance in the expected group did not decrease at the second surprise recall test after completion of the expected first recall (number of recalled word pairs; first recall: $10.10 \pm 0.69$, second recall: $10.20 \pm 0.42, p>0.85$ ). Also, performance in the unexpected group did not significantly change between both recall tests (first recall: $7.22 \pm 0.85$, second recall: $8.11 \pm 0.81, p>$ $0.07)$. Both experimental groups did not differ in the percentage change of memory performance between the first and the second recall (expected: $103.25 \pm 4.70 \%$, unexpected: $114.88 \pm 7.30 ; p>$ $0.19)$. Finally, recall performance in the expected group also remained superior to that of the unexpected group at the second recall $(p=0.03$ ). These findings are consistent with an immediate effect of retrieval expectancy on consolidation rather than retrieval, which should have equalized performance in both groups as has been consistently shown in previous studies (Goschke and Kuhl, 1993; Badets et al., 2006).

As in experiment 1, postlearning sleep in the subjects expecting the retrieval test was characterized by an increased proportion of sleep stage 4 compared with the unexpected group (11.72 \pm 1.81 vs $5.11 \pm 1.41 \%, p=0.01$; see Table 1 for a summary of sleep data). The increase was again linked to an increased power in the slow oscillation band during the first $120 \mathrm{~min}$ of non-REM sleep $(p=0.03)$, which was significant for the second and the fifth interval (e.g., for 20-40 min of non-REM sleep: $435 \pm 73$ vs $221 \pm 43, p=0.019$ ) (Fig. $2 C$ ). There was also an increased number of spindles during SWS in the expected group compared with the unexpected group ( $412 \pm 90$ vs $180 \pm 36, p=0.03$ ) (Fig. $3 C)$. In the expected group these SWS-related measures, i.e., the proportion of stage 4 sleep, the slow oscillation activity in the beginning (first $20 \mathrm{~min}$ ) of non-REM sleep, and the spindle count during SWS, each showed strong correlation with the retention of declarative memories for card pair locations (stage 4: $r=0.81$, $p=0.008$, slow oscillation activity: $r=0.67, p=0.047$, spindle count: $r=0.88, p<0.004$, respectively). In the unexpected group, corresponding correlations were generally lower and remained nonsignificant (stage 4: $r=0.61, p=0.06$, slow oscillation activity: $r=0.16, p>0.6$, spindle count: $r=-0.11, p>$ 0.78 ) (Figs. $2 D, 3 D$ ). Spindle density in SWS, as well as spindle density and spindle count in S2, was not affected by retrieval 

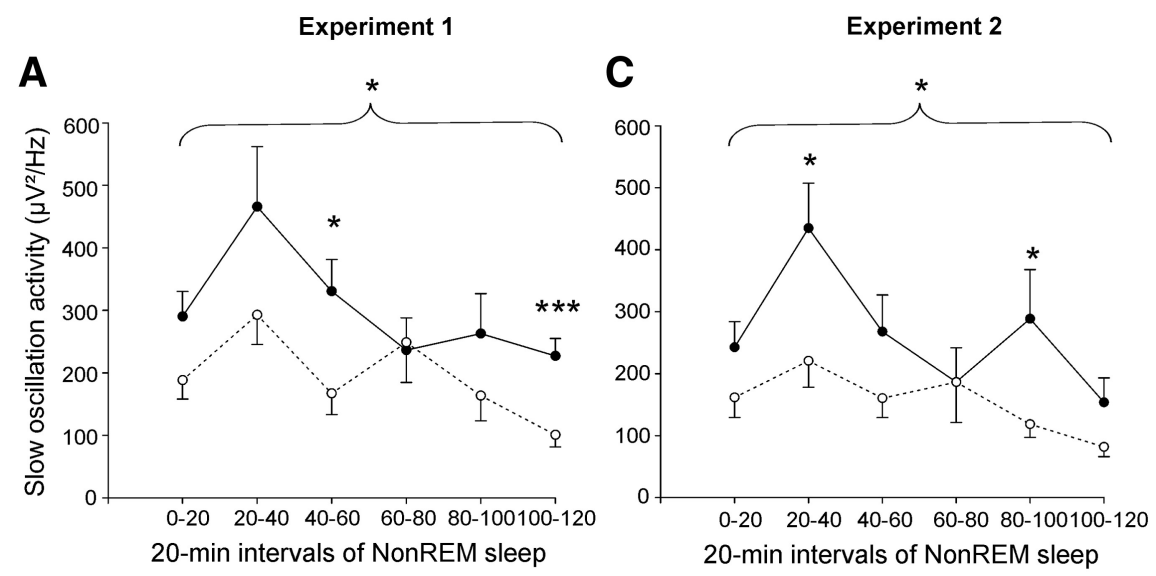

B
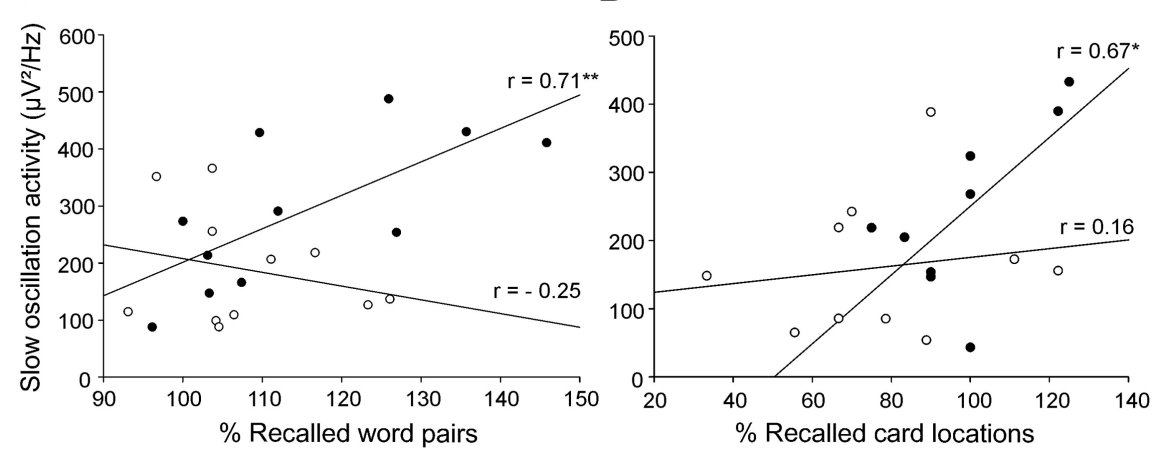

Figure 2. Relationship between retrieval expectancy, slow oscillation power, and retention of declarative memory. $\boldsymbol{A}, \boldsymbol{C}$, Mean ( \pm SEM) slow oscillation power within the first $120 \mathrm{~min}$ of non-REM sleep for six successive 20 min intervals $(0-20,20-40$ min, etc.) for subjects who expected (solid lines) and who did not expect (dotted lines) retrieval testing in experiment $1(\boldsymbol{A})$ and experiment 2 ( $C$. $\boldsymbol{B}, \boldsymbol{D}$, Correlation between slow oscillation power (during the first $20 \mathrm{~min}$ of non-REM sleep) and retention of word pairs (experiment 1) $(\boldsymbol{B})$ and card locations (experiment 2) $(\boldsymbol{D})$ separately for the subjects who expected (filled circles) and the subjects who did not expect retrieval testing (empty circles); ${ }^{*} p<0.05,{ }^{* *} p<0.01$, ${ }^{* * *} p<0.001$, for differences between groups for single $20 \mathrm{~min}$ intervals; $p<0.05$, for comparisons across the whole $120 \mathrm{~min}$ interval. Correlation coefficients are indicated. Retention performance is indicated by the percentage of recalled items (word pairs, card locations) at retrieval, with performance on the criterion trial during learning set to $100 \%$.

expectancy. None of the sleep parameters was correlated to overnight gains in procedural memory, neither in the expected group nor in the unexpected group ( $p>0.22$, for all comparisons).

\section{Discussion}

Our data show that sleep to a greater extent consolidates memories that are expected to be recalled later on and thus attain relevance for future behavior, than memories not expected to be recalled. When subjects were informed about the later retrieval test before the sleep retention interval, consolidation during sleep was enhanced in both major memory systems, i.e., for declarative memories (word pairs and card pair locations) and for procedural memories (finger tapping skills). Interestingly, though overall more variable, a sleep-associated enhancement in memory consolidation was also revealed in a subgroup of subjects who were not informed about being retested later on but nevertheless had suspected the retrieval test, suggesting that retrieval expectancy promotes sleep-dependent memory consolidation regardless of whether it is induced by instructions or spontaneously generated. The enhancing effect of retrieval expectancy was specific to sleep-dependent consolidation, because we did not observe any improvement in retention performance of the word paired associates in subjects who had been informed about the retrieval test before the wake retention intervals. Together, these behavioral findings corroborate the concept of an active consol- idation process established during sleep that, rather than globally affecting all information encoded during wakefulness, selectively strengthens those memories that are specifically linked with future expectations (Fischer and Born, 2009; Diekelmann and Born, 2010).

In our study, the expectancy of memory retrieval was induced only after the learning phase, making sure that the actual encoding depth was comparable in subjects who did or did not expect later retrieval testing, thereby excluding that the expectancy manipulation was effective already during the encoding stage of memory formation. We likewise excluded an immediate effect of retrieval expectancy on retrieval performance. It has been proposed that expectancies and intentions represent subthreshold source nodes in memory that can preactivate memory networks and thereby ease retrieval of related memories (Goschke and Kuhl, 1993; Badets et al., 2006). Contrary to this explanation, however, in our study the expectancy-induced improvement in overnight retention performance was not abolished when retrieval came as a surprise.

We carefully controlled for the possibility that subjects who expected the retrieval strengthened memories by covertly rehearsing the newly acquired memories in the interval before sleep. Data from subjects who reported engaging in any rehearsal were not included in the analyses. Whereas the verbal word pair task in experiment 1 may be easily rehearsed mentally, this is distinctly more difficult for the nonverbal $2 \mathrm{D}$ object location task in experiment 2. Finally, subjects played the computer-based game Snood after the learning phase until subjects of the sleep conditions went to bed. The highly attention-demanding nature of this game effectively prevented subjects from explicitly rehearsing any of the materials acquired previously. Even if there was some residual rehearsal activity, it is questionable whether this affected retention performance in our subjects. Previous studies failed to reveal effects of postencoding imagery of learned materials on recognition memory (Goschke and Kuhl, 1993). This agrees with our results that proved robust even when subjects reporting rehearsal were included.

Our data reveal retrieval expectancy as one important factor determining whether newly encoded memories are consolidated during sleep. This does not exclude that there are other factors that likewise facilitate access of a memory to sleep-dependent consolidation processes, such as the emotionality or salience of encoded information, which have not been manipulated here but were previously found to induce robust memory consolidation during postlearning sleep (e.g., Wagner et al., 2006; Payne et al., 2008; Wamsley et al., 2010). In a recent study, emotional memories benefited from sleep in the absence of any retrieval expectancy, whereas neutral memories did not (Payne et al., 2008). Also, the association of learned tasks with expected reward can enhance sleep-dependent memory consolidation (Fischer and Born, 
A

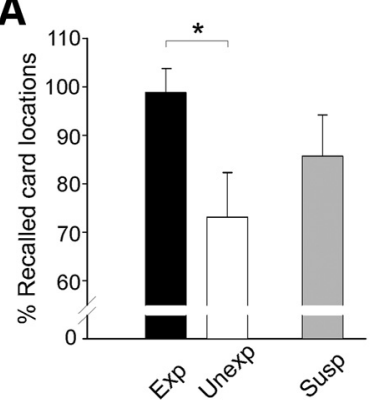

B

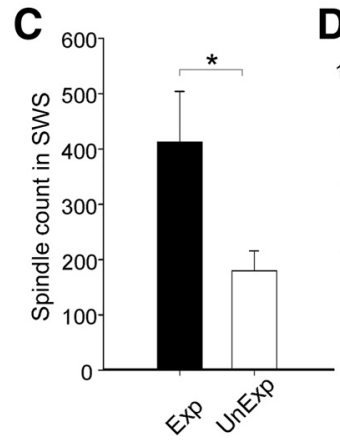

D

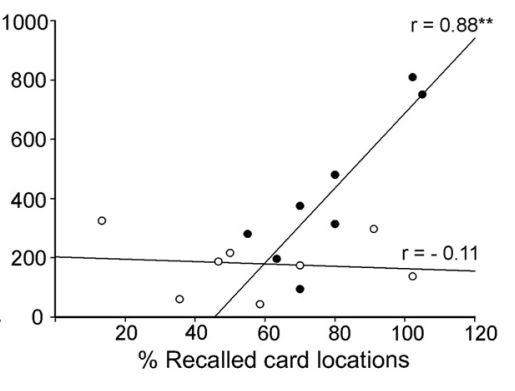

Figure 3. $\quad \boldsymbol{A}, \boldsymbol{B}$, Effects of retrieval expectancy on retention of declarative (card locations) ( $\boldsymbol{A})$ and procedural (finger sequence tapping skills) $(\boldsymbol{B})$ memories during sleep in experiment 2. Mean ( \pm SEM) retention performance is indicated for subjects who expected (Exp, black bars) and did not expect retrieval testing (Unexp, empty bars), as well as for subjects who suspected the retrieval although they were never explicitly informed about it, i.e., these subjects were originally assigned to the unexpected group (Susp, gray bars); ${ }^{*} p<0.05$, for differences between groups. Retention of card locations is indicated by the percentage of card locations recalled at retrieval testing, with performance on the criterion trial during learning set to $100 \%$. Overnight gain in finger tapping skill is indicated by the average percentage of the number of correct sequences per $30 \mathrm{~s}$ trial on the three trials at retrieval testing, with the average performance at the end of learning (trial 10-12) set to $100 \%$. C, Spindle count during SWS in the expected and unexpected groups. $D$, Correlation between spindle count during SWS and overnight retention of card locations in subjects who expected (filled circles) and who did not expect (empty circles) retrieval testing. Correlation coefficients are indicated; ${ }^{*} p<0.05$, ${ }^{* *} p<0.01$, for differences between expected and unexpected groups.

2009). The common denominator of all of these factors seems to be that they enhance the relevance of the respective information for future behavior. How these factors interact in more complex situations in real life remains to be clarified. It is likely that the brain develops a hierarchy of salience and, based on its limited processing capacity, processes only what is most salient. Such theorizing might be also of relevance for the computer game Snood, which was introduced in the present study as a distractor task but, to a certain extent, may have also been pleasant and therefore of some salience for the subjects. It might well be that the salience of Snood was in between that of the experimental task in the expected and unexpected groups, respectively, and if subjects had not played Snood immediately after the experimental task, the unexpected group might have, in fact, shown sleepdependent improvement as well. Thus, we cannot exclude that playing Snood after learning was judged more relevant than the preceding experimental learning task, especially when this learning task was not associated with future retesting (as in the unexpected group). In this case, consolidation of Snood skills would have interfered with that of the learning task in the unexpected group, whereas because of the expected retrieval in the expected group, relevance of the experimental task clearly exceeded that of Snood. Importantly however, even if Snood in this way contributed to the difference between both experimental groups, this would be quite consistent with our main conclusion that, possibly due to limited capacity, the brain during sleep preferentially consolidates relevant information.

Our data underline the importance of slow-wave sleep for consolidation of hippocampus-dependent declarative memories (Plihal and Born, 1997; Gais and Born, 2004). The enhanced memory consolidation in the subjects expecting the later retrieval test was in both experiments, with two independent subject samples, consistently linked to an increase in time spent in deepest (stage 4) SWS as well as to an increase in EEG slow oscillation activity hallmarking SWS. Note that because in our study both time in SWS and slow oscillation activity was highly correlated, both phenomena basically explain the observed memory effects equally well. In the subjects expecting retrieval, slow oscillation activity in the first $20 \mathrm{~min}$ of non-REM sleep, as well as spindle count during SWS, was significantly correlated with the overnight improvement in retention of declarative memories. The relationship between slow oscillation activity in the first 20 min and memory performance is in line with recent studies indicating a particular impact on memory processing of the early portions of slow-wave activity during the night (Huber et al., 2004, 2006, 2008). Slow oscillations are a causal factor in the consolidation of hippocampus-dependent memory (Marshall et al., 2006). Both slow oscillations and spindles are robustly associated with the retention of declarative memories (Gais et al., 2002; Mölle et al., 2004; Schabus et al., 2004; Clemens et al., 2005; Schmidt et al., 2006). Spindles nested in the depolarizing up-state of slow oscillations have been proposed as a mechanism supporting the redistribution of newly acquired declarative memories from sites of temporary storage in hippocampal circuits to sites of long-term storage within the neocortex (Marshall and Born, 2007; Diekelmann and Born, 2010). The slow oscillation up-state drives the neuronal replay of newly acquired memories in hippocampal circuits (Ji and Wilson, 2007) and thereby temporally couples hippocampal memory output toward the neocortex with the occurrence of spindles, which prime neocortical networks for the more persistent plastic changes required for the long-term storage of the memory information transferred from hippocampal sites (Rosanova and Ulrich, 2005; Diekelmann and Born, 2010). In this context, the association with SWS being less evident for the consolidation of finger tapping skills agrees well with the fact that these memories are less dependent, although not independent, of the hippocampal memory system (Schendan et al., 2003; Rauchs et al., 2008).

Importantly, a clear association between the consolidation of hippocampus-dependent memories and slow oscillation and spindle activity, respectively, was revealed only in the subjects expecting the delayed retrieval test, but not in the uninformed subjects. In fact, compared with the wake retention condition, in the uninformed subjects the sleep-dependent benefit in declarative memory consolidation failed to reach any significance, underlining that retrieval expectancy is a critical factor for the consolidation of hippocampus-dependent memory during SWS. We assume that instructing the subject after learning about the delayed retrieval forms an anticipatory context embedding the newly acquired memories into future plans. At the neuronal level, the allocation of retrieval expectancy may become manifest in a tagging of the newly encoded memory representations that facilitates their access to SWS-dependent consolidation (Marshall and Born, 2007; Fischer and Born, 2009). Processing of anticipatory aspects of behavior such as plans and expectancies is bound to prefrontal cortex functions (Miller and Cohen, 2001). Executive functions of the prefrontal cortex regulate activation of memory representations during anticipated retrieval (Cansino et al., 2002; Slotnick et al., 2003; Polyn and Kahana, 2008; Hannula and Ranganath, 2009). Pre- 
frontal tagging of hippocampal memories may likewise promote reactivation of memories during sleep-dependent consolidation, although an involvement specifically of prefrontal cortical regions cannot be inferred from our data due to the fact that we recorded EEG only from central but not from frontal electrode sites. Nevertheless, in support of this view, during postlearning SWS the neuronal assembly activity present during learning becomes replayed not only in hippocampal circuits but also in prefrontal cortex (Euston et al., 2007; Peyrache et al., 2009). We show here that sleep preferentially consolidates memories to be retrieved in the future, and the prefrontal-hippocampal system could in fact be decisive for this selectivity in memory consolidation.

\section{References}

Badets A, Blandin Y, Bouquet CA, Shea CH (2006) The intention superiority effect in motor skill learning. J Exp Psychol Learn Mem Cogn 32:491-505.

Cansino S, Maquet P, Dolan RJ, Rugg MD (2002) Brain activity underlying encoding and retrieval of source memory. Cereb Cortex 12:1048-1056.

Clemens Z, Fabó D, Halász P (2005) Overnight verbal memory retention correlates with the number of sleep spindles. Neuroscience 132:529-535.

Diekelmann S, Born J (2010) The memory function of sleep. Nat Rev Neurosci 11:114-126.

Ellenbogen JM, Payne JD, Stickgold R (2006) The role of sleep in declarative memory consolidation: passive, permissive, active or none? Curr Opin Neurobiol 16:716-722.

Euston DR, Tatsuno M, McNaughton BL (2007) Fast-forward playback of recent memory sequences in prefrontal cortex during sleep. Science 318:1147-1150.

Fischer S, Born J (2009) Anticipated reward enhances offline learning during sleep. J Exp Psychol Learn Mem Cogn 35:1586-1593.

Gais S, Born J (2004) Declarative memory consolidation: mechanisms acting during human sleep. Learn Mem 11:679-685.

Gais S, Mölle M, Helms K, Born J (2002) Learning-dependent increases in sleep spindle density. J Neurosci 22:6830-6834.

Gais S, Albouy G, Boly M, Dang-Vu TT, Darsaud A, Desseilles M, Rauchs G, Schabus M, Sterpenich V, Vandewalle G, Maquet P, Peigneux P (2007) Sleep transforms the cerebral trace of declarative memories. Proc Natl Acad Sci U S A 104:18778-18783.

Goschke T, Kuhl J (1993) Representation of intentions: persisting activation in memory. J Exp Psychol Learn Mem Cogn 19:1211-1226.

Hannula DE, Ranganath C (2009) The eyes have it: hippocampal activity predicts expression of memory in eye movements. Neuron 63:592-599.

Heine R (1914) Über Wiedererkennen und rückwirkende Hemmung. Z Psychol 68:161-236.

Huber R, Ghilardi MF, Massimini M, Tononi G (2004) Local sleep and learning. Nature 430:78-81.

Huber R, Ghilardi MF, Massimini M, Ferrarelli F, Riedner BA, Peterson MJ, Tononi G (2006) Arm immobilization causes cortical plastic changes and locally decreases sleep slow wave activity. Nat Neurosci 9:1169-1176.

Huber R, Määttä S, Esser SK, Sarasso S, Ferrarelli F, Watson A, Ferreri F, Peterson MJ, Tononi G (2008) Measures of cortical plasticity after transcranial paired associative stimulation predict changes in electroencephalogram slowwave activity during subsequent sleep. J Neurosci 28:7911-7918.

Jenkins JG, Dallenbach KM (1924) Obliviscence during sleep and waking. Am J Psychol 35:605-612.

Ji D, Wilson MA (2007) Coordinated memory replay in the visual cortex and hippocampus during sleep. Nat Neurosci 10:100-107.

Marsh RL, Hicks JL, Bink ML (1998) Activation of completed, uncompleted, and partially completed intentions. J Exp Psychol Learn Mem Cogn 24:350-361.

Marshall L, Born J (2007) The contribution of sleep to hippocampusdependent memory consolidation. Trends Cogn Sci 11:442-450.

Marshall L, Helgadóttir H, Mölle M, Born J (2006) Boosting slow oscillations during sleep potentiates memory. Nature 444:610-613.

McGaugh JL (2004) The amygdala modulates the consolidation of memories of emotionally arousing experiences. Annu Rev Neurosci 27:1-28.

Miller EK, Cohen JD (2001) An integrative theory of prefrontal cortex function. Annu Rev Neurosci 24:167-202.

Mölle M, Marshall L, Gais S, Born J (2002) Grouping of spindle activity during slow oscillations in human non-rapid eye movement sleep. J Neurosci 22:10941-10947.

Mölle M, Marshall L, Gais S, Born J (2004) Learning increases human electroencephalographic coherence during subsequent slow sleep oscillations. Proc Natl Acad Sci U S A 101:13963-13968.

Payne JD, Stickgold R, Swanberg K, Kensinger EA (2008) Sleep preferentially enhances memory for emotional components of scenes. Psychol Sci 19:781-788.

Peigneux P, Laureys S, Fuchs S, Collette F, Perrin F, Reggers J, Phillips C, Degueldre C, Del Fiore G, Aerts J, Luxen A, Maquet P (2004) Are spatial memories strengthened in the human hippocampus during slow wave sleep? Neuron 44:535-545.

Peyrache A, Khamassi M, Benchenane K, Wiener SI, Battaglia FP (2009) Replay of rule-learning related neural patterns in the prefrontal cortex during sleep. Nat Neurosci 12:919-926.

Plihal W, Born J (1997) Effects of early and late nocturnal sleep on declarative and procedural memory. J Cogn Neurosci 9:534-547.

Polyn SM, Kahana MJ (2008) Memory search and the neural representation of context. Trends Cogn Sci 12:24-30.

Rasch B, Büchel C, Gais S, Born J (2007) Odor cues during slow-wave sleep prompt declarative memory consolidation. Science 315:1426-1429.

Rauchs G, Orban P, Schmidt C, Albouy G, Balteau E, Degueldre C, Schnackers C, Sterpenich V, Tinguely G, Luxen A, Maquet P, Peigneux P (2008) Sleep modulates the neural substrates of both spatial and contextual memory consolidation. PLoS One 3:e2949.

Rechtschaffen A, Kales A (1968) A manual of standardized terminology, techniques, and scoring system for sleep stages of human subjects. Bethesda, MD: US Department of Health, Education, and Welfare-National Institutes of Health.

Rosanova M, Ulrich D (2005) Pattern-specific associative long-term potentiation induced by a sleep spindle-related spike train. J Neurosci 25:9398-9405.

Schabus M, Gruber G, Parapatics S, Sauter C, Klösch G, Anderer P, Klimesch W, Saletu B, Zeitlhofer J (2004) Sleep spindles and their significance for declarative memory consolidation. Sleep 27:1479-1485.

Schendan HE, Searl MM, Melrose RJ, Stern CE (2003) An FMRI study of the role of the medial temporal lobe in implicit and explicit sequence learning. Neuron 37:1013-1025.

Schmidt C, Peigneux P, Muto V, Schenkel M, Knoblauch V, Münch M, de Quervain DJ, Wirz-Justice A, Cajochen C (2006) Encoding difficulty promotes postlearning changes in sleep spindle activity during napping. J Neurosci 26:8976-8982.

Shimizu H (1996) Rehearsal strategies, test expectancy, and memory monitoring in free recall. Memory 4:265-287.

Slotnick SD, Moo LR, Segal JB, Hart J Jr (2003) Distinct prefrontal cortex activity associated with item memory and source memory for visual shapes. Brain Res Cogn Brain Res 17:75-82.

Szpunar KK, McDermott KB, Roediger HL 3rd (2007) Expectation of a final cumulative test enhances long-term retention. Mem Cognit 35:1007-1013.

Van Der Werf YD, Altena E, Schoonheim MM, Sanz-Arigita EJ, Vis JC, De Rijke W, Van Someren EJ (2009) Sleep benefits subsequent hippocampal functioning. Nat Neurosci 12:122-123.

Wagner U, Hallschmid M, Rasch B, Born J (2006) Brief sleep after learning keeps emotional memories alive for years. Biol Psychiatry 60:788-790.

Walker MP, Brakefield T, Morgan A, Hobson JA, Stickgold R (2002) Practice with sleep makes perfect: sleep-dependent motor skill learning. Neuron 35:205-211.

Walker MP, Brakefield T, Hobson JA, Stickgold R (2003) Dissociable stages of human memory consolidation and reconsolidation. Nature 425:616-620.

Walker MP, Stickgold R, Alsop D, Gaab N, Schlaug G (2005) Sleepdependent motor memory plasticity in the human brain. Neuroscience 133:911-917.

Wamsley EJ, Tucker M, Payne JD, Benavides JA, Stickgold R (2010) Dreaming of a learning task is associated with enhanced sleep-dependent memory consolidation. Curr Biol 20:850-855.

Wilson MA, McNaughton BL (1994) Reactivation of hippocampal ensemble memories during sleep. Science 265:676-679.

Wixted JT (2004) The psychology and neuroscience of forgetting. Annu Rev Psychol 55:235-269.

Zeigarnik B (1927) Das Behalten erledigter und unerledigter Handlungen. Psychol Forsch 9:1-85. 